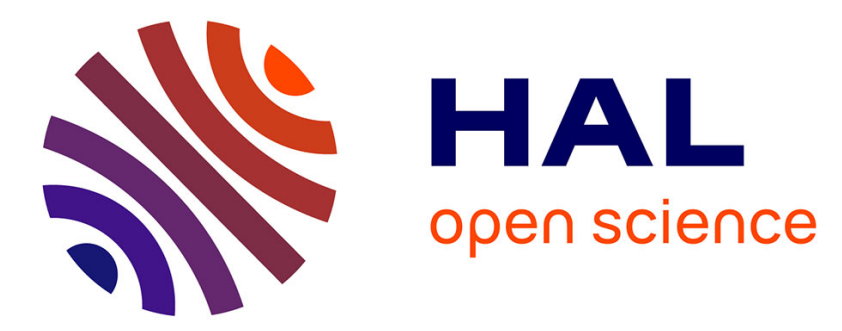

\title{
Sample Path Large Deviations for Squares of Stationary Gaussian Processes
}

Marguerite Zani

\section{To cite this version:}

Marguerite Zani. Sample Path Large Deviations for Squares of Stationary Gaussian Processes. Theory of Probability and Its Applications c/c of Teoriia Veroiatnostei i Ee Primenenie, 2012, 57 (2), pp.395405. hal-00796318

\section{HAL Id: hal-00796318 https://hal.science/hal-00796318}

Submitted on 3 Mar 2013

HAL is a multi-disciplinary open access archive for the deposit and dissemination of scientific research documents, whether they are published or not. The documents may come from teaching and research institutions in France or abroad, or from public or private research centers.
L'archive ouverte pluridisciplinaire HAL, est destinée au dépôt et à la diffusion de documents scientifiques de niveau recherche, publiés ou non, émanant des établissements d'enseignement et de recherche français ou étrangers, des laboratoires publics ou privés. 


\title{
Sample path large deviations for squares of stationary Gaussian processes
}

\author{
Marguerite Zani *
}

\begin{abstract}
In this paper, we show large deviations for random step functions of type

$$
Z_{n}(t)=\frac{1}{n} \sum_{k=1}^{[n t]} X_{k}^{2}
$$

where $\left\{X_{k}\right\}_{k}$ is a stationary Gaussian process. We deal with the associated random measures $\nu_{n}=\frac{1}{n} \sum_{k=1}^{n} X_{k}^{2} \delta_{k / n}$. The proofs require a Szegö theorem for generalized Toeplitz matrices, which is presented in the Appendix and is analogous to a result of Kac, Murdoch and Szegö [10]. We also study the polygonal line built on $Z_{n}(t)$ and show moderate deviations for both random families.
\end{abstract}

AMS classification: primary: 60G15, 60F10, 47B35 secondary: 60G10, 60G17.

Keywords: Gaussian processes, Large deviations, Szegö theorem, Toeplitz matrices.

\section{Introduction}

The aim of this paper is to provide a large deviations principle (LDP) for random functions of type

$$
Z_{n}(t)=\frac{1}{n} \sum_{k=1}^{[n t]} X_{k}^{2},
$$

and the associated polygonal line

$$
\tilde{Z}_{n}(t)=Z_{n}(t)+\left(t-\frac{[n t]}{n}\right) X_{[n t]+1}^{2},
$$

where $\left\{X_{n}\right\}_{n}$ is a stationary Gaussian process having spectral density $f$ defined on the torus $\mathbb{T}=]-\pi, \pi]$. We assume $f$ is continuous positive on $\mathbb{T}$.

Large deviations for random measures date back to Sanov [19] who showed a LDP for the family of empirical measures

$$
\frac{1}{n} \sum_{i=1}^{n} \delta_{X_{i}},
$$

*Laboratoire d'Analyse et de Mathématiques Appliquées, CNRS UMR 8050, Université Paris Est Créteil, 61 av du Gal de Gaulle, 94010, Créteil, France. e-mail: zani@u-pec.fr 
where $X_{i}$ are i.i.d. random variables.

Then, the first results on large deviations for random paths were given by Borovkov [2] and Varadhan [20]. In [2], Borovkov provides a LDP for the random polygonal line joining the points $\left(\frac{k}{n}, \frac{S_{k}}{x}\right)$ where $S_{k}=\sum_{i=1}^{k} X_{i}$ and $x=x(n)$ is in the range

$$
\limsup _{n \rightarrow \infty} \frac{x}{n}<\infty, \quad \lim _{n \rightarrow \infty} \frac{x}{\sqrt{n \ln n}}=\infty
$$

He also showed large deviations for the paths $\eta(n t) / x$ where $0 \leq t \leq 1$ and $\eta$ is a separable process with independent increments. The large deviations are given in the spaces $\mathcal{C}([0,1])$ ( the set of continuous functions on $[0,1]$ ) or $\mathcal{D}([0,1]$ ) ( the set of cadlag functions on $[0,1])$ endowed with the uniform metric. Meanwhile, Varadhan [20] proved functional large deviations in $\mathcal{D}([0,1])$ for the random step functions

$$
S_{n}(t)=\frac{1}{n} \sum_{i=1}^{[n t]} X_{i}
$$

where $t \in[0, T]$ and $[n t]$ denotes the integer part of $n t$. Later on, Mogulskii ([13]) improved these results: he proved large deviations for the polygonal line $\left(\frac{k}{n}, \frac{S_{k}}{x}\right)$ in the range

$$
\limsup _{n \rightarrow \infty} \frac{x}{n}<\infty, \quad \lim _{n \rightarrow \infty} \frac{x}{\sqrt{n}}=\infty
$$

in the space $\mathcal{D}([0,1])$ endowed with the Skorokhod metric. For more general results on large deviations for processes with independent increments, see also Lynch and Sethuraman [11], de Acosta [3] and Mogulskii [14].

The results of $[2,20,13]$ concerning step functions and continuous random polygonal lines built on sums of i.i.d. random variables can be found in the books of Dupuis and Ellis [6] and Dembo and Zeitouni [5].

In our paper, to derive the large deviations, we consider the distribution derivative of $t \rightarrow Z_{n}(t)$ and $t \rightarrow \tilde{Z}_{n}(t)$. Therefore we deal with the random measures $\nu_{n}$ and $\tilde{\nu}_{n}$ given by

$$
\left\langle\nu_{n}, h\right\rangle=\frac{1}{n} \sum_{k=1}^{n} X_{k}^{2} h\left(\frac{k}{n}\right)
$$

and

$$
\left\langle\tilde{\nu}_{n}, h\right\rangle=\sum_{k=1}^{n} X_{k}^{2} \int_{(k-1) / n}^{k / n} h(s) d s,
$$

for $h$ in $\mathcal{C}([0,1])$. Let $\mathcal{M}([0,1])$ be the set of positive bouded measures on $[0,1]$ endowed with the weak topology. Therefore $\nu_{n}$ and $\tilde{\nu}_{n}$ are a.s. in $\mathcal{M}([0,1])$.

Analogous random measures have been investigated before by Dembo and Zeitouni [4], and Gamboa and Gassiat [7]. Previous works on LDP for this kind of random functions can be found in Gamboa, Rouault and Zani [8] and Perrin and Zani [16] for stationary Gaussian processes, and in Najim [15] and Maïda, Najim and Péché [12] for 
i.i.d. sequences. We provide here a functional LDP for $\left\{\nu_{n}\right\}$ and $\left\{\tilde{\nu}_{n}\right\}$, and derive the associated LDP for $\left\{Z_{n}\right\}$ and $\left\{\tilde{Z}_{n}\right\}$. We also prove moderate deviations. The central limit theorem is known. Although part of this work was already presented in [21] the present work provide a full version with proofs and some extensions.

The remaining of the paper is organized as follows. We present in Section 2 the large and moderate deviations results. Section 3 is devoted to the proofs of Theorems. Deriving the LD result, we needed a Szegö type theorem for generalized Toeplitz matrices. This precise result is unknown to our knowledge and despite a very similar result has been shown in Kac Murdoch and Szego (see [10] and [9]), for seek of completenes we prove it in the Appendix. The remaining of the Appendix gather the proofs of technical lemmas.

\section{$2 \quad$ Large and moderate deviations}

For any $h$ in $\mathcal{C}([0,1])$, define

$\Lambda(h)=\left\{\begin{array}{l}-\frac{1}{4 \pi} \int_{[0,1]} \int_{\mathbb{T}} \log (1-2 h(t) f(\theta)) d \theta d t \quad \text { if } \forall(t, \theta) \in[0,1] \times \mathbb{T}, h(t) f(\theta)<1 / 2 \\ +\infty \quad \text { otherwise }\end{array}\right.$

Let $\Lambda^{*}$ be the Legendre dual of $\Lambda$. From Rockafellar [18], we can detail this dual function as following:

Proposition 2.1 Let $\nu$ be the measure in $\mathcal{M}([0,1])$ defined for any $h$ in $\mathcal{C}([0,1])$ by

$$
\langle\nu, h\rangle=\frac{1}{2 \pi} \int_{\mathbb{T}} f(\theta) d \theta \int_{[0,1]} h(x) d x .
$$

Let $\mu \in \mathcal{M}([0,1])$ having the following Lebesgue decomposition with respect to $\nu: \mu=$ $l \nu+\mu^{\perp}$ where $l \in \mathcal{C}([0,1])$ and $\mu^{\perp}$ is the singular part. Then

$$
\Lambda^{*}(\mu)=\int_{[0,1]} u^{*}(l(t)) \nu(d t)+\int_{[0,1]} \frac{\mu^{\perp}(d t)}{2 M},
$$

where

$$
u(x)=-\frac{1}{4 \pi} \int_{\mathbb{T}} \log (1-2 x f(\theta)) d \theta
$$

and

$$
M=\operatorname{esssup} f
$$

The function $u$ is $\mathcal{C}^{2}$ on $(-\infty, 1 / 2 M)$, and

$$
\begin{array}{r}
u^{\prime}(x)=\frac{1}{2 \pi} \int_{\mathbb{T}} \frac{f(\theta)}{1-2 x f(\theta)} d \theta \\
u^{\prime \prime}(x)=\frac{1}{\pi} \int_{\mathbb{T}} \frac{f(\theta)^{2}}{(1-2 x f(\theta))^{2}} d \theta>0
\end{array}
$$


Hence $u^{\prime}$ is strictly increasing, and $\lim _{x \rightarrow-\infty} u^{\prime}(x)=0$. On the other hand, we denote $u^{\prime}(1 / 2 M):=\lim _{x \rightarrow+\infty} u^{\prime}(x) \leq+\infty$ (e.g. if $\left.f \in \mathcal{C}^{2}, u^{\prime}(1 / 2 M)=+\infty\right)$. The recession function ( see Theorem 8.5 of [18]) is $r\left(u^{*} ; y\right)=y / 2 M$.

\subsection{Large Deviations}

We can now state the LDP result:

Theorem 2.2 The families $\left\{\nu_{n}\right\}_{n \in \mathbb{N}}$ and $\left\{\tilde{\nu}_{n}\right\}_{n \in \mathbb{N}}$ satisfy a LDP in $\mathcal{M}([0,1])$ with speed $n$ and rate function $\Lambda^{*}$.

We can carry the previous LDP to the random functions $Z_{n}$ and $\tilde{Z}_{n}$. Following Lynch and Sethuraman [11] and de Acosta [3], we introduce some notations. Let $D([0,1], \mathbb{R})$ be the space of cadlag real functions on $[0,1]$, and $b v([0,1], \mathbb{R}) \subset D([0,1], \mathbb{R})$ the space of bounded variation functions. We can identify $b v([0,1], \mathbb{R})$ with $\mathcal{M}([0,1])$ : to $h$ in $b v([0,1], \mathbb{R})$ corresponds $\mu_{h}$ in $\mathcal{M}([0,1])$ characterized by $\mu_{h}([0, t])=h(t)$. Up to this identification, the topological dual of $b v([0,1], \mathbb{R})$ is the set $\mathcal{C}([0,1])$. We endow $b v([0,1], \mathbb{R})$ with the $w^{*}$-topology written $\sigma$, i.e. the topology induced by $\mathcal{C}([0,1])$ on $\mathcal{M}([0,1])$. Now, let us define the rate function associated to $Z_{n}$ and $\tilde{Z}_{n}$ : let $h$ be in $b v([0,1], \mathbb{R})$ and $\mu_{h}$ the associated measure in $\mathcal{M}([0,1])$; let $\mu_{h}=\left(\mu_{h}\right)_{a}+\left(\mu_{h}\right)_{s}$ be the Lebesgue decomposition of $\mu_{h}$ in absolutely continuous and singular terms with respect to the Lebesgue measure on $[0,1]$; let $h_{a}(t)=\left(\mu_{h}\right)_{a}([0, t])$ and $h_{s}(t)=\left(\mu_{h}\right)_{s}([0, t])$. Set

$$
\Phi(h)=\int_{[0,1]} u^{*}\left(h_{a}^{\prime}\right)(t) \nu(d t)+r h_{s}(1),
$$

where $u^{*}$ and $r$ are defined in Proposition 2.1.

Theorem 2.3 The families of random functions $\left\{Z_{n}\right\}$ and $\left\{\tilde{Z}_{n}\right\}$ satisfy a LDP on the space $(b v([0,1], \mathbb{R}), \sigma)$, with speed $n$ and rate function $\Phi$.

\section{$2.2 \quad$ Moderate deviations}

We can state also in this case a moderate deviation principle. We detail it for $\nu_{n}$, it is the same for $\tilde{\nu}_{n}$. Let $\left\{a_{n}\right\}$ be a sequence of positive real numbers such that $a_{n} \rightarrow 0$ and $n a_{n} \rightarrow+\infty$ when $n \rightarrow+\infty$. Set

$$
Y_{n}=\sqrt{n a_{n}}\left(\nu_{n}-E\left(\nu_{n}\right)\right) .
$$

We have the following moderate deviations principle

Theorem $2.4\left\{Y_{n}\right\}$ satisfy a LDP with speed $a_{n}^{-1}$ and good rate function defined, for all $\mu \in \mathcal{M}([0,1])$ by

$$
I(\mu)=\left\{\begin{array}{l}
\frac{\pi}{2 \bar{f}^{2}} \int_{[0,1]} l(x)^{2} d x \text { if } \mu(d x)=l(x) d x \\
+\infty \text { otherwise, }
\end{array}\right.
$$

where

$$
\bar{f}^{2}=\frac{1}{2 \pi} \int_{\mathbb{T}} f^{2}
$$




\subsection{Generalizations}

The previous results can be generalized to some other random functions.

\subsubsection{Weighted random variables}

Assume $g$ is a continuous function on $[0,1]$ and define

$$
W_{n}=\frac{1}{n} \sum_{k=1}^{[n t]} g\left(\frac{k}{n}\right) X_{k}^{2}
$$

For any $h$ in $\mathcal{C}([0,1])$, define

$\Lambda(h)=\left\{\begin{array}{l}-\frac{1}{4 \pi} \int_{[0,1]} \int_{\mathbb{T}} \log (1-2 h(t) g(t) f(\theta)) d \theta d t \quad \text { if } \forall(t, \theta) \in[0,1] \times \mathbb{T}, h(t) g(t) f(\theta)<1 / 2 \\ +\infty \text { otherwise }\end{array}\right.$

The previous large deviations results apply with rate function $\Lambda^{*}$.

\subsubsection{Quadratic forms built on the stationary process}

We define

$$
m=\operatorname{essinf} f
$$

and assume $m>0$. Let $F$ be a continuous positive function on $[m, M]$. Let $O$ be an orthonormal matrix such that $O^{*} T_{n}(f) O$ is the diagonal matrix whose $i$-th diagonal element is $\mu_{i, n}$ the $i$-th eigenvalue of $T_{n}(f)$. Define

$$
F\left(T_{n}(f)\right)=O D_{f} O^{*}
$$

where $D_{f}$ is the diagonal matrix whose $i$-th element is $F\left(\mu_{i, n}\right)$. Define the following quadratic form

$$
W_{n}=\frac{1}{n} X^{*} F\left(T_{n}(f)\right) X=\frac{1}{n} Y^{*} Y,
$$

where $Y=\left(Y_{1}, \cdots Y_{n}\right)$ is the vector defined by

$$
Y=F\left(T_{n}(f)\right)^{1 / 2} X .
$$

In this case, $W_{n}$ satisfies a LDP and moderate deviations theorem with rate function $\Lambda^{*}$ where for any $h$ in $\mathcal{C}([0,1])$

$\Lambda(h)= \begin{cases}-\frac{1}{4 \pi} \int_{[0,1]} \int_{\mathbb{T}} \log [1-2 h(t) f(\theta) F[f(\theta)]] d \theta d t \quad \text { if } \forall(t, \theta) \in[0,1] \times \mathbb{T}, h(t) f(\theta)<1 / 2 \\ +\infty \quad \text { otherwise }\end{cases}$ 


\section{Proof of the large and moderate deviations}

We first give some asymptotic properties for the families $\left\{\nu_{n}\right\}_{n}$ and $\left\{\tilde{\nu}_{n}\right\}_{n}$.

\subsection{Weak convergence of $\nu_{n}$ and $\left\{\tilde{\nu}_{n}\right\}_{n}$}

Lemma 3.1 Let $h$ be in $\mathcal{C}([0,1])$.

$$
\left\langle\nu_{n}, h\right\rangle \rightarrow\langle\nu, h\rangle \quad \text { in probability } \quad \text { as } n \rightarrow+\infty
$$

and

$$
\left\langle\tilde{\nu}_{n}, h\right\rangle \rightarrow\langle\nu, h\rangle \quad \text { in probability } \quad \text { as } n \rightarrow+\infty
$$

where

$$
\langle\nu, h\rangle=\bar{f} \int_{[0,1]} h(x) d x
$$

and

$$
\bar{f}=\frac{1}{2 \pi} \int_{\mathbb{T}} f(\theta) d \theta
$$

Proof:

Let $h$ be in $\mathcal{C}([0,1])$, and consider

$$
\left\langle\nu_{n}, h\right\rangle=\frac{1}{n} \sum_{k=1}^{n} X_{k}^{2} h\left(\frac{k}{n}\right) .
$$

Set $X$ the Gaussian vector $\left(X_{1}, X_{2}, \cdots, X_{n}\right)$ and $\Delta_{h}$ the diagonal matrix

$$
\left(\begin{array}{cccc}
h\left(\frac{1}{n}\right) & 0 & 0 & 0 \\
0 & h\left(\frac{2}{n}\right) & 0 & 0 \\
0 & 0 & \ddots & 0 \\
0 & 0 & 0 & h(1)
\end{array}\right)
$$

Therefore we can write

$$
\left\langle\nu_{n}, h\right\rangle=\frac{1}{n} X^{*} \Delta_{h} X,
$$

where $X^{*}$ denote the transpose of $X$. By an orthonormal change of basis,

$$
\left\langle\nu_{n}, h\right\rangle=\frac{1}{n} U_{n}^{*} T_{n}(f)^{1 / 2} \Delta_{h} T_{n}(f)^{1 / 2} U_{n},
$$

where $U_{n}$ is a standard normal vector and $T_{n}(f)$ the order- $n$ Toeplitz matrix associated to $f$. Therefore

$$
\left\langle\nu_{n}, h\right\rangle=\frac{1}{n} \sum_{k=1}^{n} \lambda_{k, n} Z_{k, n}
$$


where $\left\{Z_{k, n}\right\}$ are independent $\chi^{2}(1)$-distributed random variables, and $\left\{\lambda_{k, n}\right\}$ are the eigenvalues of $T_{n}(f)^{1 / 2} \Delta_{h} T_{n}(f)^{1 / 2}$.

We can write as well

$$
\left\langle\tilde{\nu}_{n}, h\right\rangle=\frac{1}{n} \sum_{k=1}^{n} \tilde{\lambda}_{k, n} Z_{k, n}
$$

where $\left\{Z_{k, n}\right\}$ are independent $\chi^{2}(1)$-distributed random variables, and $\left\{\tilde{\lambda}_{k, n}\right\}$ are the eigenvalues of $T_{n}(f)^{1 / 2} A_{h} T_{n}(f)^{1 / 2}$, and the matrix $A_{h}$ is diagonal with $k$-th diagonal term

$$
\left(A_{h}\right)_{k, k}=\int_{(k-1) / n}^{k / n} h(s) d s .
$$

We have the two following results on the distributions $\left\{\lambda_{k, n}\right\}$ and $\left\{\tilde{\lambda}_{k, n}\right\}$, which proofs are postponed to the Appendix.

Lemma 3.2 The sequences $\left\{\lambda_{k, n}\right\}$ and $\left\{\tilde{\lambda}_{k, n}\right\}$ are bounded as follows:

$$
\forall n \in \mathbb{N}, \forall 1 \leq k \leq n, \quad \begin{aligned}
\left|\lambda_{k, n}\right| & \leq\|h\|_{\infty}\|f\|_{\infty} \\
& \left|\tilde{\lambda}_{k, n}\right| \leq\|h\|_{\infty}\|f\|_{\infty}
\end{aligned}
$$

Lemma 3.3 For any $p$ in $\mathbb{N}, p \geq 1$,

$$
\begin{aligned}
\lim _{n \rightarrow+\infty} \frac{1}{n} \sum_{k=1}^{n} \lambda_{k, n}^{p} & =\frac{1}{2 \pi} \int_{[0,1]} \int_{\mathbb{T}}(h(t) f(\theta))^{p} d t d \theta . \\
\lim _{n \rightarrow+\infty} \frac{1}{n} \sum_{k=1}^{n}\left(\tilde{\lambda}_{k, n}\right)^{p} & =\frac{1}{2 \pi} \int_{[0,1]} \int_{\mathbb{T}}(h(t) f(\theta))^{p} d t d \theta .
\end{aligned}
$$

With the above lemma,

$$
\lim _{n \rightarrow+\infty} E\left(\left\langle\nu_{n}, h\right\rangle\right)=\langle\nu, h\rangle .
$$

Moreover,

$$
\lim _{n \rightarrow+\infty} n \operatorname{Var}\left\langle\nu_{n}, h\right\rangle=\frac{2}{n} \sum_{k=1}^{n} \lambda_{k, n}^{2}=\frac{1}{\pi} \int_{[0,1]} \int_{\mathbb{T}}(h(t) f(\theta))^{2} d t d \theta .
$$

We do as well for $\tilde{\nu}_{n}$, and it ends the proof of lemma 3.1.

\subsection{Proof of Theorem 2.2:}

The proof follows exactly the scheme [8]. We detail here for $\nu_{n}$, it is similar for $\tilde{\nu}_{n}$. With the decomposition (11), we get the n.c.g.f. of $\nu_{n}$ : for any $h \in \mathcal{C}([0,1])$,

$$
\Lambda_{n}(h)=\frac{1}{n} \log E\left(\exp \left\{n\left\langle\nu_{n}, h\right\rangle\right\}\right)= \begin{cases}-\frac{1}{2 n} \sum_{k=1}^{n} \log \left(1-2 \lambda_{k, n}\right) & \text { if } \forall k, \lambda_{k, n}<1 / 2 \\ +\infty & \text { otherwise }\end{cases}
$$

From Lemma 3.3, we can determine the limit of $\Lambda_{n}$ in two cases: 
- if $\forall(t, \theta) \in[0,1] \times \mathbb{T} \quad h(t) f(\theta)<1 / 2$, then

$$
\lim _{n \rightarrow+\infty} \Lambda_{n}(h)=-\frac{1}{4 \pi} \int_{[0,1]} \int_{\mathbb{T}} \log (1-2 h(t) f(\theta)) d \theta d t=\Lambda(h) .
$$

- if $\exists(t, \theta) \in[0,1] \times \mathbb{T} ; \quad h(t) f(\theta)>1 / 2$, then for $n$ large enough, $\Lambda_{n}(h)=+\infty$ and

$$
\lim _{n \rightarrow+\infty} \Lambda_{n}(h)=+\infty=\Lambda(h) .
$$

These two cases do not cover the whole set $\mathcal{C}([0,1])$. Nevertheless, this will be sufficient for the LDP, since they contain a dense subset of exposing hyperplanes of $\Lambda^{*}$.

Upper bound

From Theorem 4.5.3 b) of [5], and the following lemma, which proof is postponed to the Appendix, the upper bound holds for compact sets.

Lemma 3.4 For any $\delta>0$ and $\mu$ in $\mathcal{M}([0,1])$, there exists $h_{\delta}$ in $\mathcal{C}([0,1])$ such that:

$$
\begin{array}{r}
\forall(t, \theta), h_{\delta}(t) f(\theta)<1 / 2 \\
\int_{[0,1]} h_{\delta}(t) d \mu(t)-\Lambda\left(h_{\delta}\right) \geq \Lambda_{\delta}^{*}(\mu)
\end{array}
$$

where

$$
\Lambda_{\delta}^{*}(\mu)=\min \left\{\Lambda^{*}(\mu)-\delta, \frac{1}{\delta}\right\}
$$

\section{Exponential tightness}

Remark that for a real number $a$,

$$
\left\{\sup _{\|h\|_{\infty} \leq 1}\left\langle\nu_{n}, h\right\rangle \geq a\right\} \subset\left\{\nu_{n}(1) \geq a\right\} .
$$

If $M=\operatorname{esssup}_{\theta} f(\theta)$, for any $y<1 / 2 M$,

$$
\limsup _{n} \frac{1}{n} \log P\left(\nu_{n}(1) \geq a\right) \leq-y a-\frac{1}{4 \pi} \int_{[0,1]} \int_{\mathbb{T}} \log (1-2 y f(\theta)) d \theta,
$$

and

$$
\lim _{a \rightarrow+\infty} \limsup _{n} \frac{1}{n} \log P\left(\nu_{n}(1) \geq a\right)=-\infty .
$$

Hence the sequence $\left(\nu_{n}\right)$ is exponentially tight, and the upper bound holds for any closed set of $\mathcal{M}([0,1])$.

\section{Lower bound}

We study the set of exposed points of $\Lambda^{*}$ (see [5]). Let

$$
\mathcal{H}=\left\{\mu \in \mathcal{M}([0,1]) ; \mu=l \nu, 0<l<u^{\prime}(1 / 2 M), l \text { continuous on }[0,1]\right\} .
$$

The following two lemmas, which proofs are postponed to the Appendix, show that that $\mathcal{H}$ is a dense subset of the exposed points of $\Lambda^{*}$, which concludes the proof of Theorem 2.2 . 
Lemma 3.5 Let $\mu=l \nu$ be in $\mathcal{H}$. There exists $h_{l}$ in $\mathcal{C}([0,1])$ such that

$$
\begin{array}{r}
\forall(t, \theta) \in[0,1] \times \mathbb{T} \quad h_{l}(t) f(\theta)<1 / 2 \\
\forall \xi \in \mathcal{M}([0,1]) \quad \Lambda^{*}(\mu)-\Lambda^{*}(\xi)<(\mu-\xi)\left(h_{l}\right)
\end{array}
$$

Furthermore, there exists $\gamma>1$ such that $\Lambda(\gamma l)<+\infty$.

Hence $\mu$ is an exposed point of $\Lambda^{*}$ with exposing hyperplane $h_{l}$.

Lemma 3.6 Let $\mu$ be in $\mathcal{M}([0,1])$ such that $\Lambda^{*}(\mu)<+\infty$. There exists a sequence $\left(\mu_{n}\right) \in \mathcal{H}$ such that $\mu_{n} \Rightarrow \mu$ and $\lim _{n \rightarrow+\infty} \Lambda^{*}\left(\mu_{n}\right)=\Lambda^{*}(\mu)$.

\subsection{Proof of Theorem 2.4:}

The n.c.g.f. of $Y_{n}$ is given for any $h$ in $\mathcal{C}[m, M]$ by

$$
\begin{aligned}
\Lambda_{n}(h) & =a_{n} \log E\left(\exp \left\{\sqrt{\frac{n}{a_{n}}}\left(\left\langle\nu_{n}, h\right\rangle-E\left(\left\langle\nu_{n}, h\right\rangle\right)\right)\right\}\right) \\
& =-\frac{a_{n}}{2} \sum_{k=1}^{n} \log \left(1-\frac{2}{\sqrt{n a_{n}}} \lambda_{k, n}\right)+\frac{2}{\sqrt{n a_{n}}} \lambda_{k, n}
\end{aligned}
$$

We recall that $\left\{\lambda_{k, n}\right\}$ are the eigenvalues of the matrix $T_{n}(f)^{1 / 2} \Delta_{h} T_{n}(f)^{1 / 2}$. We can assert

$$
\Lambda_{n}(h)=\frac{1}{n} \sum_{k=1}^{n} \lambda_{k, n}^{2}+O\left(\frac{1}{n \sqrt{n a_{n}}} \sum_{k=1}^{n}\left|\lambda_{k, n}\right|^{3}\right) .
$$

From the convergence (10), Therefore

$$
\lim _{n \rightarrow+\infty} \Lambda_{n}(h)=\Lambda=\bar{f}^{2} \int_{[0,1]} h(x)^{2} d x
$$

This function is defined on all $\mathcal{C}[0,1]$, then the rate function is the Legendre dual of $\Lambda$ which is, from Rockafellar [18],

$$
I(\mu)=\frac{\pi}{2 \bar{f}^{2}} \int_{[0,1]} l(x)^{2} d x,
$$

where $d_{\mu}(t)=l(x) d x$.

\section{Appendix}

\subsection{A Szegö Theorem for generalized Toeplitz matrices}

In this paragraph we show a result on the distribution of eigenvalues of some kind of generalized Toeplitz matrices. 
Suppose $g$ is a real function defined on $[0,1] \times \mathbb{T}$ such that for any $x \in[0,1], g(x, \cdot) \in$ $L^{1}(\mathbb{T})$. Define

$$
\hat{g}_{k}(x)=\frac{1}{2 \pi} \int_{\mathbb{T}} g(x, \theta) e^{-i k \theta} d \theta
$$

and

$$
T_{n}^{\mathrm{gen}}(g)_{k, l}=\hat{g}_{l-k}\left(\frac{k}{n}\right)
$$

Denote by

$$
\left\|\hat{g}_{k}\right\|_{\infty}=\sup _{x \in[0,1]}\left|\hat{g}_{k}(x)\right|
$$

Theorem 4.1 Under assumption

$$
\begin{gathered}
M:=\sum_{k}\left\|\hat{g}_{k}\right\|_{\infty}<\infty \\
\lim _{n \rightarrow \infty} \frac{1}{n} \operatorname{tr}\left(T_{n}^{g e n}(g)\right)^{p}=\frac{1}{2 \pi} \int_{0}^{1} \int_{\mathbb{T}} g(x, \theta)^{p} d \theta d x .
\end{gathered}
$$

Proof: This proof is analogous to the one of [10]. Let $\varepsilon>0$ be fixed and $m \in \mathbb{N}$ chosen such that:

$$
\sum_{|k|>m}\left\|\hat{g}_{k}\right\|_{\infty}<\varepsilon
$$

Consider the trigonometric polynom of degree $m$ :

$$
g^{m}(x, \theta)=\sum_{k=-m}^{m} \hat{g}_{k}(x) e^{i k \theta}
$$

Let $T_{n}^{\text {gen }}\left(g^{m}\right)$ be the generalized Topelitz matrix associated to $g^{m}$ as in (17). Therefore

$$
T_{n}^{\mathrm{gen}}(g)=T_{n}^{\mathrm{gen}}\left(g^{m}\right)+R
$$

and the sum of the moduli of the elements of any row of $R$ is less than $\varepsilon$. Hence the same is true for the eigenvalues of $R$ i.e. for the eigenvalues of $T_{n}^{\text {gen }}(g)-T_{n}^{\text {gen }}\left(g^{m}\right)$. From the Weyl-Courant Lemma, we can therefore bound

$$
\left|\lambda_{k, n}-\lambda_{k, n}^{m}\right| \leq \varepsilon
$$

where $\left\{\lambda_{k, n}\right\}_{k}$ and $\left\{\lambda_{k, n}^{m}\right\}_{k}$ are the eigenvalues of $T_{n}^{\text {gen }}(g)$ and $T_{n}^{\text {gen }}\left(g^{m}\right)$ respectively nondecreasingly ordered. From assumption (18),

$$
\left|\lambda_{k, n}\right| \leq M, \quad\left|\lambda_{k, n}^{m}\right| \leq M
$$

Hence for any positive integer $s$

$$
\left|\left(\lambda_{k, n}\right)^{s}-\left(\lambda_{k, n}^{m}\right)^{s}\right| \leq \varepsilon s M^{s-1} .
$$


We can bound similarly $\left|g(x, \theta)^{s}-g^{m}(x, \theta)^{s}\right|$ and therefore to show (19) it is enough to consider the polynomial $g^{m}$. We derive

$$
\lim _{n \rightarrow \infty} \frac{1}{n} \operatorname{tr}\left(T_{n}^{\mathrm{gen}}\left(g^{m}\right)\right)^{p}=\sum_{D_{p}} \sum_{j=1}^{m} \hat{g}_{l_{1}}\left(\frac{j+l_{1}}{n}\right) \hat{g}_{l_{2}}\left(\frac{j+l_{1}+l_{2}}{n}\right) \cdots \hat{g}_{l_{p}}\left(\frac{j}{n}\right)
$$

where $D_{p}=\left\{\left(l_{1}, \cdots l_{p}\right) \in \mathbb{Z}^{p} ; \sum l_{i}=0\right\}$ and the second sum in the RHS above is on $j$ such that $j+\sum_{1}^{k} l_{i}$ - for $k$ from 1 to $p$ - is in the range $1, \ldots, n$, i.e. $s p \leq j \leq n-s p$. Therefore we have to suppress at most $2 s p+1$ terms. From classical results on Riemann sums,

$$
\begin{array}{r}
\lim _{n \rightarrow \infty} \frac{1}{n} \sum_{D_{p}} \sum_{j=1}^{m} \hat{g}_{l_{1}}\left(\frac{j+l_{1}}{n}\right) \hat{g}_{l_{2}}\left(\frac{j+l_{1}+l_{2}}{n}\right) \cdots \hat{g}_{l_{p}}\left(\frac{j}{n}\right) \\
=\sum_{D_{p}} \int_{0}^{1} \hat{g}_{l_{1}}(x) \hat{g}_{l_{2}}(x) \cdots \hat{g}_{l_{p}}(x) d x \\
=\sum_{\left(l_{1}, \cdots l_{p}\right) \in \mathbb{Z}^{p}} \frac{1}{2 \pi} \int_{\mathbb{T}} e^{i\left(l_{1}+l_{2}+\cdots l_{p}\right)} d \theta \int_{0}^{1} g_{l_{1}}(x) \hat{g}_{l_{2}}(x) \cdots \hat{g}_{l_{p}}(x) d x \\
=\frac{1}{2 \pi} \int_{0}^{1} \int_{\mathbb{T}} g(x, \theta)^{p} d \theta d x .
\end{array}
$$

\subsection{Proof of Proposition 2.1}

This lemma is a consequence of Theorem 5 of Rockafellar [18]. For the sake of clarity, we recall the framework of that paper. Let $h$ be in $\mathcal{C}([m, M])$, and

$$
\Lambda(h)=\int_{[m, M]} u(t, h(t)) d \nu(t),
$$

where $u(t, x)$ defined on $[m, M] \times \mathbb{R} \rightarrow \mathbb{R}$ is a function convex in $x$, and $\nu$ a nonnegative, $\sigma$-finite measure. For any $\mu$ in $\mathcal{M}([m, M])$ having, with respect to $\nu$ the Lebesgue decomposition $\mu=l \nu+\mu^{\perp}$, where $l \in \mathcal{C}([m, M])$, and $\mu^{\perp}$ is the singular part, then

$$
\Lambda^{*}(\mu)=\int_{[m, M]} u^{*}(t, l(t)) d \nu(t)+\int_{[m, M]} r\left(u^{*}(t, \cdot) ; d \mu^{\perp} / d \eta(t)\right) d \eta(t)
$$

where $\eta$ is any nonnegative measure of $\mathcal{M}([m, M])$ with respect to which $\mu^{\perp}$ is absolutely continuous, and $u^{*}(t, \cdot)$ is the dual function of $u(t, \cdot)$ :

$$
\forall t, \quad u^{*}(t, y)=\sup _{x \in \mathbb{R}}\{x y-u(t, x)\} .
$$

Applying the result of $(21)$ to $u(t, x)=-(1 / t) \log (1-2 t x)$, we have the formula of Proposition 2.1 


\subsection{Proof of Lemma 3.2}

From Proposition V 1.8 and Theorem X 1.1 of Bhatia [1], since $T_{n}(f)$ is an hermitian positive matrix,

$$
\left\|T_{n}(f)^{1 / 2} \Delta_{h} T_{n}(f)^{1 / 2}\right\| \leq\left\|T_{n}(f)\right\|\left\|\Delta_{h}\right\|
$$

From Grenander and Szegö ([9] p.64)

$$
\left\|T_{n}(f)\right\| \leq\|f\|_{\infty} .
$$

In addition,

$$
\left\|\Delta_{h}\right\| \leq \sup _{k} \sum_{s}\left|\left(\Delta_{h}\right)_{k s}\right| \leq\|h\|_{\infty}
$$

Getting together inequalities (22) and (23), we get the result.

\subsection{Proof of Lemma 3.3}

This lemma is a direct consequence of Theorem 4.1 above, for both random measures.

\subsection{Proof of Lemma 3.4}

From the definition of $\Lambda^{*}$, for any $\delta>0$, there exists $h_{\delta}$ in $\mathcal{C}([0,1])$ such that inequality (14) holds. In case we only have

$$
\forall(t, \theta) \in[0,1] \times \mathbb{T} \quad h_{\delta}(t) f(\theta) \leq \frac{1}{2},
$$

we choose $h_{\varepsilon}$ with $\varepsilon>0$ such that

$$
\int_{[0,1]} h_{\varepsilon}(t) d \mu(t)-\Lambda\left(h_{\varepsilon}\right) \geq \Lambda_{\delta}^{*}(\mu)-\varepsilon .
$$

(this is possible from the continuity of $\Lambda$ in a neighborhood of $h_{\delta}$ )

Then (14) holds with another $\delta$. From assumption on $f, f>0$, then $h_{\varepsilon} f<1 / 2$.

\subsection{Proof of Lemma 3.5}

For all $0<y<1 / u^{\prime}(1 / 2 M)$, there exists a unique $x_{y}$ in $(-\infty, 1 / 2 M)$ such that $y=$ $u^{\prime}\left(x_{y}\right)$. For such a pair $\left(y, x_{y}\right)$,

$$
u^{*}(y)=y x_{y}-u\left(x_{y}\right) .
$$


Since $u^{\prime}$ is strictly increasing and continuous, $u^{*}$ is strictly convex on $0<y<u^{\prime}(1 / 2 M)$. For such an $y$ and $z>0, z \neq y$,

$$
u^{*}(y)-u^{*}(z)<(y-z) x_{y}
$$

(then $y$ is an exposed point of $u^{*}$ with exposing hyperplane $x_{y}$ ) If $\mu=l \nu$ and $\xi=\tilde{l} \nu+\xi^{\perp}$. We apply inequality (24) with $y=l(t)$ and $z=\tilde{l}(t)$, and then we integrate over $[0,1]$ against $\nu$. We obtain the inequality (15) with $h_{l}(t)=x_{l(t)}$.

\subsection{Proof of Lemma 3.6}

Following the sketch of proof of [8], we proceed in 4 steps. Assume $u^{\prime}(1 / 2 M)=+\infty$.

Step 1: Let $\mu=l \nu+\mu^{\perp}$ be in $\mathcal{M}([0,1])$ such that $\Lambda^{*}(\mu)<\infty$ with $l$ continuous and $l \in \overline{\left(0, u^{\prime}\left(\frac{1}{2 M}\right)\right.}$, and such that $\mu^{\perp}$ is in $L^{1}([0,1])$. Since $\nu$ has full support on $[0,1]$, there exists a sequence of continuous positive functions on $[0,1]$ such that $h_{n} d \nu \Rightarrow \mu^{\perp}$. From the lower semi-continuity of $\Lambda^{*}$,

$$
\liminf _{n \rightarrow+\infty} \Lambda^{*}\left(\left(l+h_{n}\right) \nu\right) \geq \Lambda^{*}(\mu) .
$$

Since $u^{*}$ is a convex function, from Rockafellar (see [17]), for any $y>0$ and $z \geq 0$,

$$
u^{*}(y+z) \leq u^{*}(y)+\frac{z}{2 M} .
$$

Therefore

$$
\Lambda^{*}((l+\tilde{l}) \nu) \leq \Lambda^{*}(l \nu)+\frac{1}{2 M} \int \tilde{l}(t) d \nu(t)
$$

From inequality above,

$$
\Lambda^{*}\left(\left(l+h_{n}\right) \nu\right) \leq \Lambda^{*}(l \nu)+\frac{1}{2 M} \int_{0,1]} h_{n} d \nu
$$

And then

$$
\liminf _{n \rightarrow+\infty} \Lambda^{*}\left(\left(l+h_{n}\right) \nu\right) \leq \Lambda^{*}(\mu) .
$$

We now show that the Lemma is true if $\mu=l \nu$ with $l \nu$-a.s. in $\left(0, u^{\prime}\left(\frac{1}{2 M}\right)\right.$ and integrable.

\section{Step 2}

We prove the result for $\mu=l \nu$ assuming that $l$ is in $\left(0, u^{\prime}\left(\frac{1}{2 M}\right)\right.$ integrable and that for some $\epsilon>0, l>\epsilon \nu$-a.s. There exists a sequence $\left(l_{n}\right)$ of continuous positive functions such that $l_{n}$ converges both in $L^{1}(\nu)$ norm and $\nu$-a.s. to $l$ and $l_{n}>\epsilon / 2$. Since on $\left(\epsilon / 2, u^{\prime}\left(\frac{1}{2 M}\right)\right.$ the function $u^{*}$ is Lipschitzian, the lemma holds.

$\underline{\text { Step } 3}$ 
Define $l_{\epsilon}:=l 1_{l>\epsilon}+\epsilon 1_{l \leq \epsilon}$. Apply second step and inequality (25) noticing that $l_{\epsilon}$ converges in $L^{1}(\nu)$ to $l$ and that $l_{\epsilon} \geq l$.

Step 4

For $\mu=l \nu+\eta$, combine first and third step.

If $u^{\prime}(1 / 2 M)<+\infty$, we have to modify the second and third step, introducing an additional truncation at level $u^{\prime}(1 / 2 M)-\varepsilon$.

\section{References}

[1] Bhatia, R. Matrix analysis. Graduate Texts in Mathematics, 169, Springer, New York, 1996.

[2] Borovkov, A. A. Boundary value problems for random walks and large deviations in function spaces. Teor. Verojatnost. i Primenen, 12, pp 635-654, 1967.

[3] de Acosta, A. Large deviations for vector-valued Lévy processes. Stoch. Proc. Appl., 51, pp 75-115, 1994.

[4] Dembo, A. and Zeitouni, O. Large deviations for subsampling from individual sequences. Stat. and Prob. Lett., 27, pp 201-205, 1996.

[5] Dembo, A. and Zeitouni, O. Large deviations techniques and applications (second edition). Springer ,1998.

[6] P. Dupuis and R.S. Ellis A weak convergence approach to the theory of large deviations. Wiley Series in Probability and Statistics, Wiley, 1997.

[7] Gamboa, F. and Gassiat, E. Bayesian methods for ill posed problems. Annals of Stat, 25, pp 328-350, 1997.

[8] Gamboa, F., Rouault, A. and Zani, M. A functional large deviations principle for quadratic forms of Gaussian stationary processes. Stat. and Prob. Letters,43, pp299-308, 1999.

[9] Grenander, U. and Szegö, G. Toeplitz forms and their applications. University of California Press, 1958.

[10] Kac, M. , Murdock, W. L. and Szegö, G. On the eigenvalues of certain hermitian forms. Journal of Rat. Mech. and An., 2, pp 767-800,1953.

[11] J. Lynch and J. Sethuraman Large deviations for processes with independent increments. Ann. of Probab., 15, pp 610-627,1987.

[12] Maïda, M., Najim, J. and Péché, S. Large deviations for weighted empirical mean with outliers. Stochastic Process. Appl.,117, pp 1373-1403, 2007.

[13] Mogulskii, A.A. Large deviations for trajectories of multi dimensional random walks. Th. Prob. Appl., 21, pp 300-315, 1976.

[14] Mogulskii, A.A. Large deviations for processes with independent increments. Ann. of Prob.., 21, pp 202-215, 1993.

[15] Najim, J. A Cramér type theorem for weighted random variables. Electronic Journ. of Prob.,7, pp 1-32, 2002. 
[16] Perrin, O. and Zani, M. Large deviations for sample paths of Gaussian processes quadratic variations. Zap. Nauchn. Sem. S.-Peterburg. Otdel. Mat. Inst. Steklov. (POMI) transl. in J. Math. Sci. (N. Y.), 328 (transl. in 139), pp 169-181 (transl. 6595-6602), 2005 (transl. in 2006).

[17] Rockafellar, R. T. Convex analysis. Princeton University Press, 1970.

[18] Rockafellar, R. T. Integrals which are convex functionals II. Pac. Journ. of Maths, 39, pp 439-469, 1971.

[19] Sanov, I.N. On the probability of large deviations of random magnitudes. Math. Sb. N. S., 42(84), pp 11-44, 1957.

[20] Varadhan, S. R. S. Asymptotic probabilities and differential equations.. Comm. Pure Appl. Math., 19, pp 261-286, 1966.

[21] Zani, M. Grandes déviations pour des fonctionnelles issues de la statistique des processus Thèse, Orsay, 2000. 